\title{
Actin-based motility propelled by molecular motors
}

\author{
Sai Pramod Upadyayula $\cdot$ Murali Rangarajan
}

Received: 30 November 2011 / Accepted: 6 March 2012/Published online: 27 March 2012

(c) The Author(s) 2012. This article is published with open access at Springerlink.com

\begin{abstract}
Actin-based motility of Listeria monocytogenes propelled by filament end-tracking molecular motors has been simulated. Such systems may act as potential nanoscale actuators and shuttles useful in sorting and sensing biomolecules. Filaments are modeled as three-dimensional elastic springs distributed on one end of the capsule and persistently attached to the motile bacterial surface through an end-tracking motor complex. Filament distribution is random, and monomer concentration decreases linearly as a function of position on the bacterial surface. Filament growth rate increases with monomer concentration but decreases with the extent of compression. The growing filaments exert push-pull forces on the bacterial surface. In addition to forces, torques arise due to two factors-distribution of motors on the bacterial surface, and coupling of torsion upon growth due to the right-handed helicity of F-actin - causing the motile object to undergo simultaneous translation and rotation. The trajectory of the bacterium is simulated by performing a force and torque balance on the bacterium. All simulations use a fixed value of torsion. Simulations show strong alignment of the filaments and the long axis of the bacterium along the direction of motion. In the absence of torsion, the bacterial surface essentially moves along the direction of the long axis. When a small amount of the torsion is applied to the
\end{abstract}

S. P. Upadyayula

Department of Electronics and Communication Engineering,

Amrita Vishwa Vidyapeetham, Coimbatore, India

M. Rangarajan ( $\square)$

Department of Chemical Engineering and Materials Science,

Amrita Vishwa Vidyapeetham, Coimbatore, India

e-mail: r_murali@cb.amrita.edu bacterial surface, the bacterium is seen to move in righthanded helical trajectories, consistent with experimental observations.

Keywords Actin-based motility - Molecular motor . Simulation $\cdot$ Listeria

\section{Introduction}

Actin-based motility of Listeria monocytogenes has been studied extensively as a model problem to help uncover the biochemical and biophysical mechanisms of cell motility. There are some amazing similarities between the motility of Listeria and a wide variety of biological systems such as motility of Shigella, nematode sperm motility, and chromosome alignment and locomotion in mitosis (Dickinson and Purich 2002). There are also major similarities in the biochemical and biophysical mechanisms between the motility of Listeria and intracellular actin-propelled cell crawling. Such systems act as potential nanoscale actuators and shuttles useful in sorting and sensing biomolecules. In these systems, propulsive forces and rotating torques are generated at the polymerizing end of actin filaments anchored to the bacterial surface by end-tracking proteins, propelled by ATP hydrolysis, and energy of the so-called treadmilling (depolymerization at cytosolic end and polymerization at the motile end) (Pantaloni et al. 2001; Pollard and Borisy 2003; Carlier et al. 2003; Borisy and Svitkina 2000). Some features of actin-based motility are brought out in Fig. 1. Figure 1a illustrates the so-called treadmilling of actin filaments in the cell. Figure $1 \mathrm{~b}$ identifies the biochemical players in recruiting actin treadmilling for the propulsion of a motile surface (Listeria or cell membrane). Despite research for over four decades, a number of 


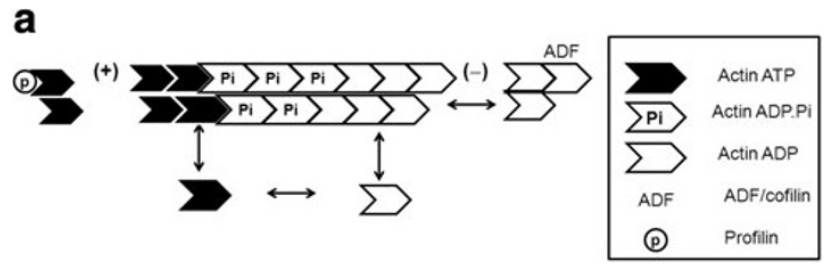

b

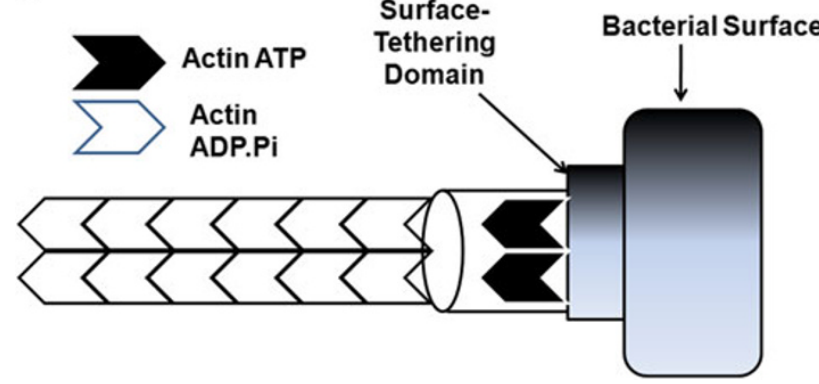

Fig. 1 a Treadmilling of actin filaments; polymerization happens at the $(+)$ end or the barbed end while depolymerization happens at the $(-)$ end or the pointed end. The process is catalyzed by a number of proteins, including profilin $(\mathrm{P})$ which catalyzes monomer addition and ATP/ADP exchange, and ADF/cofilin (ADF) which catalyzes depolymerization at the $(-)$ ends. b Actin-based propulsion of a motile surface; the key assumption is that the filaments are tethered to the motile surface through the surface-tethering domain. In this work, we have assumed that this domain acts as a hinge holding an elastic spring (filament) to the surface. Through the surface-tethering domain, the filaments exert force and torque on the motile surface, in response to which the surface translates and rotates

questions remain unanswered in the broad problem of cell motility and in the specific problems of actin-based motility of Listeria and of cell crawling. Of particular interest for the current work are the following:

- How is the energy available from treadmilling and ATP hydrolysis converted into forces and torques necessary for the propulsion and rotation of the bacterium?

- Why are long-length scale helical trajectories observed in Listeria motility (Robbins and Theriot 2003; Zeile et al. 2005)?

Such questions are fundamental in providing the first step towards designing actuators and sensors using these motors, in designing better scaffolds in tissue engineering, and in the discovery of next-generation medicines.

In the present work, starting from a mechanochemical model based on affinity-modulated processive elongation of actin filaments by molecular motors (Dickinson et al. 2004), actin-based motility is simulated for a model capsule-shaped bacterium. Using appropriate system and process parameters, the trajectories of the bacterium in the presence and absence of torsion are examined. The possible origin of right-handed helical trajectories observed with bacteria is discussed.

\section{Models of actin-based motility}

Three classes of models have been proposed that seek to explain force generation in these systems. In elastic propulsion-type models (Noireaux et al. 2000; Gerbal et al. 2000; Paluch et al. 2006, for instance), the pushing forces are generated by a network of growing filaments on the curved motile surface. Circumferential stresses mount upon barbed-end growth until a critical point is reached where local gel fracture occurs and the object is thrust forward. The second class of models is the so-called Brownian-ratchet model (Peskin et al. 1993; Mogilner and Oster 1996, 2003; Mogilner 2006; Lin 2009; Lin et al. 2010), where the force is generated by the Brownian motion of the polymerizing filaments. Monomer addition takes place during the monomer-sized excursions of the barbed ends from the motile surface. In consistent with experimental advances that have established filament-surface attachment and the role of Arp $2 / 3^{1}$ complex in filament nucleation, Mogilner and Oster (2003) and Mogilner (2006) have amended their elastic Brownian ratchet model to a tethered ratchet model, which employs two types of filaments, those that are detached, undergo elongation and generate force and those that undergo nucleation by surface-bound Arp 2/3 complex, which is also tethered to their 'mother' filaments already linked to the cytoskeletal network. Such a mechanism generates push-pull forces until the Arp 2/3 units detach from the motile surface, at which point they become 'working' filaments that generate forces to propel the surface. There are a number of issues with this model, including the fact that it does not consistently account for the binding of actin monomers by $\operatorname{VASP}^{2}$ (Kühnel et al. 2004), and that it is unable to consistently account for the torsion due to growth, which might be responsible for the observed longlength scale rotation of Listeria (Robbins and Theriot 2003; Zeile et al. 2005). A recent paper by Dayel et al. (2009) combines these two mechanisms and explains symmetry breaking in motility. An in silico reconstitution of Listeria motility has been presented recently (Alberts and Odell 2004), where the nano-saltation of Listeria as observed by Kuo and McGrath (2000) is reproduced. However, there is no explicit accounting of torsion due to monomer addition, in this work. The third class of models, proposed by Dickinson and Purich $(2002,2006)$, Dickinson et al. (2004) and Dickinson (2008), considers motility effected by affinity-modulated processive motors. The present work, belonging to this class, models bacterial motility as being facilitated by motor complexes localized on the motile surface, which tether the growing actin

\footnotetext{
${ }^{1}$ This complex is responsible for filament branching.

${ }^{2}$ A surface-localized protein capable of binding actin monomers.
} 
filaments and transmits the forces and torques due to filament elongation to the motile surface. A brief discussion of this model is provided in below.

\section{Actin-based motility propelled by molecular motors}

\section{Rate of filament growth}

The mechanistic model for actin-based motility, a modified version of what was originally proposed by Dickinson and Purich (2002), Dickinson et al. 2004, and Dickinson (2008), is essentially as follows. It is known that surface proteins such as VASP and $\operatorname{ActA}^{3}$ get localized at the motile surface during actin-based motility (Cameron et al. 1999). One of such proteins is proposed to be part of a motor complex that tethers actin filaments. The motor complex is proposed to have higher affinity for ATP-actin ${ }^{4}$ as opposed to ADP-actin. ATP-actin, usually associated with profilin, ${ }^{5}$ binds at the growing end of an existing filament, which is held by the motor complex. ATP hydrolysis results in the penultimate unit becoming ADPactin for which the motor complex has less affinity. This results in the shifting and rebinding of the motor complex to the newly bound ATP-actin unit, thus completing the cycle. The rate of monomer addition is proportional to the concentration of the monomers available in the region as well as the force that the compressive or tensile filament is subjected to due to confinement between the bacterial surface and its anchor at the other end in the cytoskeletal network. A concentration gradient exists between the bulk cytosol and the center of the filament bundle propelling the bacterium, and is approximated in this work as a linear profile, with the bulk concentration set equal to the experimentally measured critical concentration of actin monomer at the growing end. As the filament is compressed more, it resists further growth, as captured in the following equation (Dickinson 2008):

$r(F)=\frac{k_{0} C}{1+\frac{k_{0} C}{k_{t, 0} \exp (-F d / k T)}}$

In the above equation, $r$ indicates the rate of growth of a compressed filament, $k_{0}$ is the rate constant for monomer binding, the term $k_{\mathrm{t}, 0} \exp [-F d / k T]$ estimates the resistance to filament growth due to the compressive force that the

\footnotetext{
${ }^{3}$ VASP is present in eukaryotic cells, whereas ActA is present in Listeria, binds to VASP, which in turn is known to bind actin monomers.

${ }^{4}$ Monomeric actin is usually affiliated with either ATP or ADP; it is known that ATP affiliated with actin hydrolyzes during actin treadmilling.

5 A small protein in the solution, localized at the region of motility, is known to bind actin and facilitate filament elongation in treadmilling.
}

filament is subjected to, with $F$ denoting the compressive force. This force-dependence is incorporated through a transition state model, with the barrier presumed to be located at $d=2.7 \mathrm{~nm}$.

\section{Forces on filaments}

As discussed above, the filament elongation rate is force dependent. To capture this, the filament is modeled as a three-dimensional elastic spring, described by the following equations, taken from Dickinson (2008):

$\boldsymbol{F}_{i}(\boldsymbol{r})=-\boldsymbol{K}_{i} \cdot\left[\boldsymbol{r}_{i}-\left(\boldsymbol{r}_{0, i}+\boldsymbol{n}_{i} L\right)\right]$,

where, $\boldsymbol{r}_{0, i}$ is the filament end position at the cytoskeletal matrix, $L$ is the length of the filaments (with the assumption that treadmilling keeps the length at a steady value of $100 \mathrm{~nm}), \boldsymbol{r}_{i}$ is the filament end position at the growing end, and $\boldsymbol{n}_{i}$ is the tangent vector at the growing end. A compressive filament is assumed to be bent with a constant curvature given by the tangent vector $\boldsymbol{n}_{i}$. All filament forces are much less than the buckling force, which is about $35 \mathrm{pN}$ for actin. Therefore the filaments have no radii of curvature, and $\boldsymbol{n}_{i}$ merely is the unit vector along the line connecting the two filament ends. A tensile filament results when the end position is such that the filament is stretched, and its orientation is straight along the $\boldsymbol{n}_{\boldsymbol{i}}$ it had when it was previously compressive. The tensile spring constant is taken to be $60 \mathrm{pN} / \mathrm{nm}$, while the compressive stiffness tensor and the change in filament orientation after each growth are defined according to Dickinson (2008). When the bacterial surface translates or rotates due to applied forces and/or torques, the filament-end position at the surface changes, thereby changing $\boldsymbol{n}_{i}$.

\section{Bacterial position and orientation}

The bacterium is tracked through a position vector $R$, assigned to the center of the circular plane joining the back hemispherical portion of the capsule with its cylindrical portion. The orientation of the bacterium is tracked through three angles, denoted as $\alpha, \beta$, and $\gamma$, with respect to the $x y$, $y z$, and $z x$ planes, respectively. The distribution of the motor complexes on the bacterial surface is modeled by considering coaxial circular planes of varying radii from zero (containing the central tensile filament) to the bacterial radius, with randomly assigned number of motor complexes on the circumference of each circle.

Torques on filaments

Finally, the filament growth applies torque on the bacterium due to two factors: 
$\boldsymbol{T}=\sum_{i}\left(\boldsymbol{r}_{i} \times \boldsymbol{F}_{i}\right)+\boldsymbol{\tau}_{i}$.

The first term in the right-hand side of the above equation is a positional torque due to the position of the filament ends (and motors) away from the point on the bacterium whose position is assigned as its position. The second term is a parameter representing the torsion resulting from addition of a monomer unit to a righthanded helical filament. In the absence of information to rigorously model the addition of torsion and the mechanisms of releasing it, a small fixed torsion $\tau$ is imposed on the surface for each filament growth. The ability of the surface to rotate and balance this torsion is examined. In this work, we do not consider the possibility of the filament detaching from the surface. A proper modeling of filament detachment requires accounting for filament branching and possible reattachment. We leave this to a future work. After each set of growths, the forces and torques on the filaments are balanced by suitably translating and rotating the bacterium. Thus, our simulations are not, in a true sense, dynamic. There is an inherent assumption that the growth of the filaments occurs at a time scale longer than the effects of drag forces. At molecular length scales, this is generally a questionable assumption. Dickinson (2008), and Dickinson and Purich (2002) have simulated the system by incorporating drag forces, to which our results are similar, indicating the processive nature of the motor complex and its ability to keep the filaments tethered throughout. This seems to, in effect, damp the effects of drag. We present our results with a caveat that this assumption has to be tested further with a more comprehensive model.

\section{Results and discussion}

The parameters used in the simulations are listed in Table 1. Most of these parameters are chosen along similar lines of arguments presented in Dickinson and Purich (2002), and Dickinson (2008). Figure 2 shows the rate of filament growth as a function of the compressive force it is subject to. The results, similar to those obtained in Dickinson (2008), indicate two features: for small forces, the rate of filament elongation is insensitive to forces, whereas the growth rate drops to nearly zero at a stall force of about $18-20 \mathrm{pN}$. The sensitivity of the growth rate to force may be tuned by simply tuning the parameter $k_{\mathrm{t}, 0}$, which indicates the growth rate of an filament subject to no force. Figure 3 indicates the concentration profile of actin monomer at various points on the motile surface, considered in the simulations. While it is possible to simulate the gradient more rigorously (Dickinson 2008), only a linear
Table 1 Parameters used in simulations

\begin{tabular}{|c|c|c|}
\hline Symbol & Model parameter & Value used \\
\hline$C_{\infty}$ & Bulk monomer concentration & $4 \mu \mathrm{M}$ \\
\hline$d$ & $\begin{array}{l}\text { Filament length increase due } \\
\text { to monomer addition }\end{array}$ & $2.7 \mathrm{~nm}$ \\
\hline$\kappa_{\mathrm{f}, \perp}$ & Spring constant of compressive filament & $0.124 \mathrm{pN} / \mathrm{nm}$ \\
\hline$\kappa_{\mathrm{f}, \|}$ & Spring constant of compressive filament & $0.041 \mathrm{pN} / \mathrm{nm}$ \\
\hline$\kappa_{\mathrm{t}}$ & Spring constant of tensile filament & $60 \mathrm{pN} / \mathrm{nm}$ \\
\hline$k_{0}$ & Monomer growth rate constant & $10 / \mu \mathrm{M} / \mathrm{s}$ \\
\hline$k_{\mathrm{t}, 0}$ & Monomer transfer rate constant & $10^{4} / \mathrm{s}$ \\
\hline$L$ & Uncross-linked filament length & $100 \mathrm{~nm}$ \\
\hline$L_{\mathrm{s}}$ & Bacterial side length & $800 \mathrm{~nm}$ \\
\hline$R$ & Bacterial radius & $400 \mathrm{~nm}$ \\
\hline$\tau$ & $\begin{array}{l}\text { Torsion added to each filament due } \\
\text { to monomer addition }\end{array}$ & $10.8 \mathrm{pN} / \mathrm{nm}$ \\
\hline
\end{tabular}

Source: Dickinson (2008)

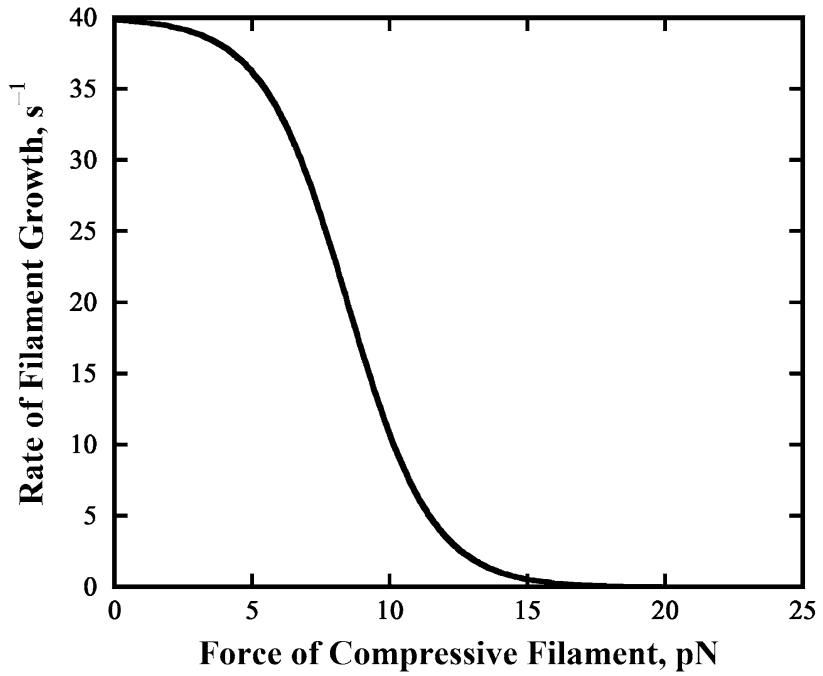

Fig. 2 Rate of filament elongation as a function of compressive force

gradient profile is chosen in this work. The concentration gradients, along with motor complex distribution on the surface, cause varying growth rates, and are responsible for symmetry breaking in motility and the resulting trajectories. A simulated trajectory of the bacterium is shown in Fig. 4, where the torsion due to monomer addition is not considered. The forces and torques are balanced before each growth. It is seen that the direction of motion quickly aligns itself along the long axis of the bacterium, with the central tensile filament also assuming the same orientation. If the motor complex distribution on the surface is not random but skewed-see, for instance, Rafelski and Theriot (2006) and Shaevitz and Fletcher (2008)—-this could introduce an additional mechanism for symmetry breaking, which is not examined in this study. 


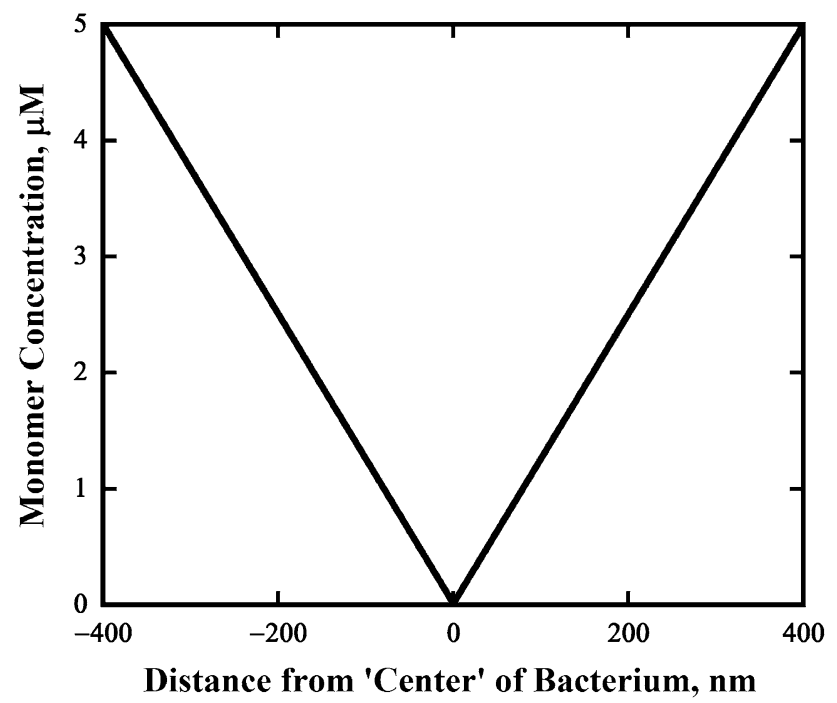

Fig. 3 Concentration gradient of the actin-ATP monomer from the bulk cytosol to the center of the actin tail; this gradient is approximated to be a linear function in this work. More sophisticated models have been developed (Dickinson 2008). However, the key physics remains unaltered

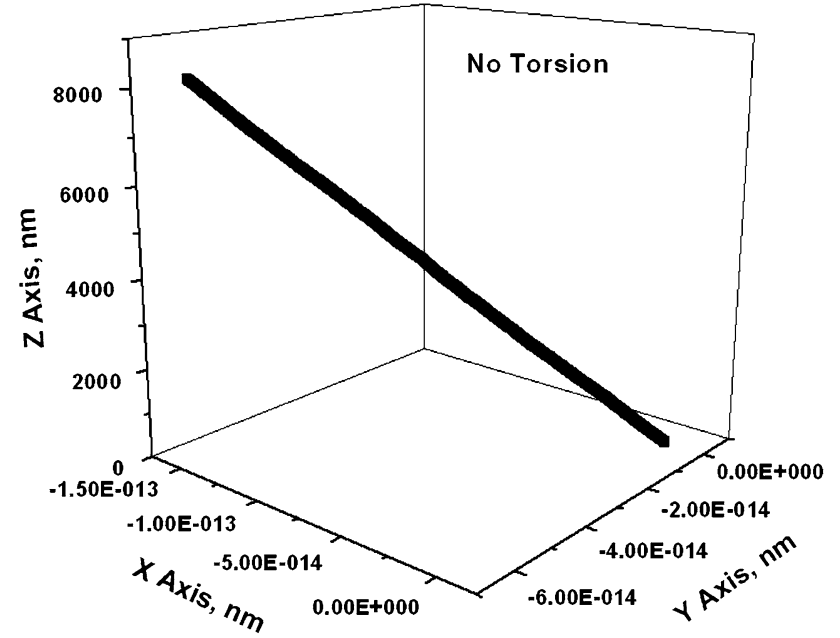

Fig. 4 Trajectory of the bacterium in the absence of torsion on the filament from monomer addition; after an initial alignment of the filaments towards the long axis of the bacterium, the bacterium essentially proceeds along the direction of its long axis

We now consider the effect of torsion due to monomer addition on motility by adding a small torsion of $\tau=10.8 \mathrm{pN} / \mathrm{nm}$ to each filament upon monomer addition. There is little clarity as to the exact mechanisms in which torsion due to monomer addition might be released. In the absence of details, $\tau$ is simply treated as a parameter to indicate the effect of releasing the torsion by rotational motion of the bacterial surface. It is seen from the resultant trajectory, Fig. 5, that torsion due to monomer addition may bias the trajectory to be a right-handed helical

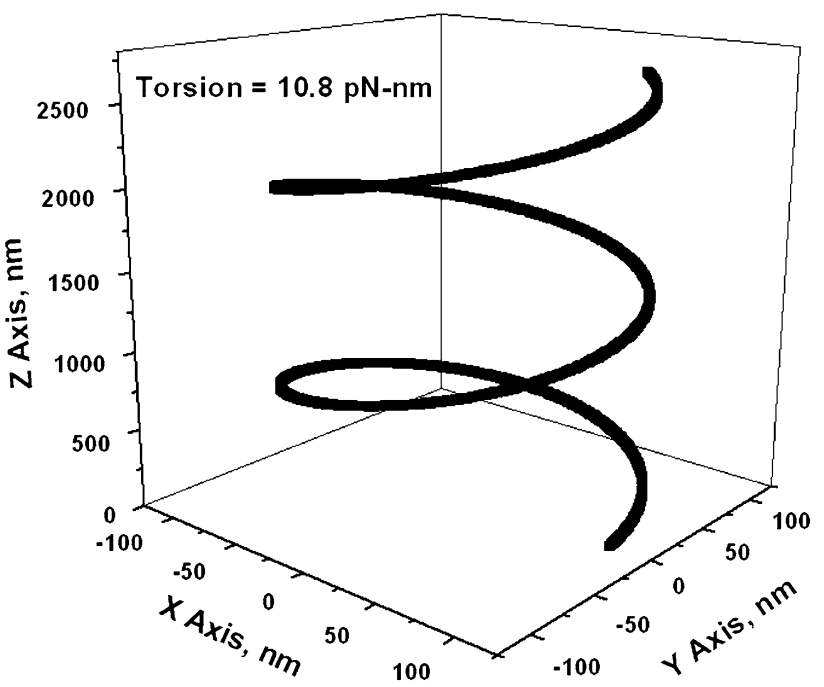

Fig. 5 Trajectory of the bacterium with torsion on the filament due to monomer addition $(10.8 \mathrm{pN} / \mathrm{nm})$; torsion breaks symmetry in the trajectory and causes right-handed helical trajectories of the bacterium

trajectory. The helical trajectory in this simulation has a radius of about $0.14 \mu \mathrm{m}$ and pitch of about $1.2 \mu \mathrm{m}$. Thus, experimentally observed right-handed helical trajectories may be explained by the partial release of torsion due to filament growth by the rotation of the surface. On the other hand, if filament torsion becomes an unimportant parameter in simulations (this may be due to filament detachment or network/motor compliance), the trajectories lose the persistent helical shape and become saltatory.

\section{Conclusions}

Actin-based motility of L. monocytogenes is simulated based on the biophysical mechanism of affinity-modulated processive elongation of tethered filaments to motor complexes distributed on the bacterial surface. The motor complexes and the anchor points of the filaments to the actin network at their pointed end are treated as partially compliant. When torsion due to monomer addition is not considered, strong alignment of the trajectory with the long axis of the model capsule is observed. Upon consideration of torsion due to monomer addition, symmetry breaking arises, resulting in right-handed helical trajectories.

Open Access This article is distributed under the terms of the Creative Commons Attribution License which permits any use, distribution, and reproduction in any medium, provided the original author(s) and the source are credited.

\section{References}

Alberts JB, Odell GM (2004) In silico reconstitution of Listeria propulsion exhibits nano-saltation. PLoS Biol 2:2054-2066

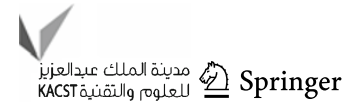


Borisy GG, Svitkina TM (2000) Actin machinery: pushing the envelope. Curr Opin Cell Biol 12:104-112

Cameron LA, Footer MJ, van Oudenaarden A, Theriot JA (1999) Motility of ActA protein-coated microspheres driven by actin polymerization. Proc Natl Acad Sci USA 96:4908-4913

Carlier MF, Le Clainche C, Wiesner S, Pantaloni D (2003) Actinbased motility: from molecules to movement. BioEssays 25:336-345

Dayel MJ, Akin O, Landeryou M, Risca V, Mogilner A, Mullins RD (2009) In silico reconstitution of actin-based symmetry breaking and motility. PLoS Biol 7:1-21

Dickinson RB (2008) A multi-scale mechanistic model for actinpropelled bacteria. Cell Mol Bioeng 1:110-121

Dickinson RB, Purich DL (2002) Clamped-filament elongation model for actin-based motors. Biophys J 82:605-617

Dickinson RB, Purich DL (2006) Diffusion rate limitations in actinbased propulsion of hard and deformable particles. Biophys $\mathrm{J}$ 91:1548-1563

Dickinson RB, Caro L, Purich DL (2004) Force generation by cytoskeletal filament end-tracking proteins. Biophys J 87:28382854

Gerbal F, Chaikin P, Rabin Y, Prost J (2000) An elastic analysis of Listeria monocytogenes propulsion. Biophys J 79:2259-2275

Kuo SC, McGrath JL (2000) Steps and fluctuations of Listeria monocytogenes during actin-based motility. Nature 407:10261029

Kühnel K, Jarchau T, Wolf E, Schlichting I, Walter U, Wittinghofer A, Strelkov SV (2004) The VASP tetramerization domain is a right-handed coiled coil based on a 15-residue repeat. Proc Natl Acad Sci USA 101:17027-17032

Lin Y (2009) Mechanics model for actin-based motility. Phy Rev E 79:021916
Lin Y, Shenoy VB, Hu B, Bai L (2010) A microscopic formulation for the actin-driven motion of Listeria in curved paths. Biophys J 99:1043-1052

Mogilner A (2006) On the edge: modeling protrusion. Curr Opin Cell Biol 18:32-39

Mogilner A, Oster GF (1996) Cell motility driven by actin polymerization. Biophys J 71:3030-3045

Mogilner A, Oster GF (2003) Force generation by actin polymerization: the elastic ratchet and tethered filaments. Biophys $\mathrm{J}$ 84:1591-1605

Noireaux V, Golsteyn RM, Friederich E, Prost J, Antony C, Louvard D, Sykes C (2000) Growing an actin gel on spherical surfaces. Biophys J 78:1643-1654

Paluch E, van der Gucht J, Joanny J-F, Sykes C (2006) Deformation in actin comets from rocketing beads. Biophys J 91:3113-3122

Pantaloni D, Le Clainche C, Carlier MF (2001) Mechanism of actinbased motility. Science 292:1502-1506

Peskin CS, Odell GM, Oster GF (1993) Cellular motions and thermal fluctuations: the Brownian ratchet. Biophys J 65:316-324

Pollard TD, Borisy GG (2003) Cellular motility driven by assembly and disassembly of actin filaments. Cell 112:453-465

Rafelski SM, Theriot JA (2006) Mechanism of polarization of Listeria monocytogenes surface protein ActA. Mol Microbiol 59:1262-1279

Robbins JR, Theriot JA (2003) Listeria monocytogenes rotates around its long axis during actin-based motility. Curr Biol 13:R754-R756

Shaevitz JW, Fletcher DA (2008) Curvature and torsion in growing actin networks. Phys Biol 5:026006

Zeile WL, Zhang F, Dickinson RB, Purich DL (2005) Listeria's righthanded helical rocket-tail trajectories: mechanistic implications for force generation in actin-based motility. Cell Motil Cytoskeleton 60:121-128 